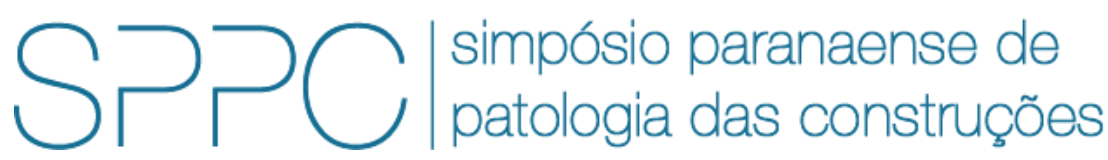

ISSN 2526-7248 artigo 2SPPC1010, pp. 103-116, 2017

\title{
Avaliação de ensaios não destrutivos para a detecção de concretos dosados com diferentes teores de contaminação por íons sulfeto
}

\author{
Ana Paula B. Capraro1, Júlio Cesar P. Frandoloso², Marcelo Henrique F. Medeiros ${ }^{3}$, Mariana D. G. \\ Portella ${ }^{4}$, Kleber F. Portella ${ }^{4}$, Bruna Godke ${ }^{5}$ e Isabela Oliveira ${ }^{5}$. \\ 1 Doutoranda, Universidade Federal do Paraná, anapcapraro@gmail.com \\ 2 Graduando, Universidade Federal do Paraná, juliofrandoloso@gmail.com \\ 3 Professor Doutor, Universidade Federal do Paraná, medeiros.ufpr@gmail.com \\ 4 Doutor(a), Institutos LACTEC, mariana.portella@lactec.org.br; portella@lactec.org.br \\ ${ }^{5}$ Mestre, COPEL, bruna.godke@copel.com; isabela.oliveira@copel.com
}

\begin{abstract}
Resumo: Este estudo avaliou o emprego de ensaios não destrutivos, ultrassom e resistividade elétrica, na diferenciação de compostos contaminados por íons sulfeto em três idades, 84, 168 e 360 dias. Para tal análise foram moldadas quatro séries de concreto, sendo uma a referência, sem contaminação, e as demais com uma substituição parcial do agregado miúdo por pirita $(0,38 \%, 0,75 \%$ e $3,75 \%)$. Os teores de contaminação da mistura foram definidos a partir de regulamentações normativas, as quais limitam o teor de $\mathrm{SO}_{3}$ com relação à massa total dos agregados do composto. Para favorecer as reações de hidratação, optou-se pela permanência dos corpos de prova, até os 28 dias, em cura submersa com água saturada com cal. Após esse período, os materiais foram submetidos a um mecanismo de envelhecimento acelerado, no qual os corpos de prova foram alternados semanalmente entre um tanque com solução aquosa, continuamente aerada, e uma câmara seca, ambos com temperatura e umidade controladas. Dentre os resultados obtidos neste estudo, somente o ensaio de resistência à compressão axial apresentou redução do desempenho na série de maior contaminação. As séries intermediárias não apresentaram desempenho inferior quando comparadas à série referência. Este resultado corrobora com o proposto nas recomendações normativas, as quais permitem a utilização de 0,5\% (ACl) e 1,0\% (AFNOR) de $\mathrm{SO}_{3}$. Concluiu-se, ainda, que, para o período e método de envelhecimento propostos, os ensaios não destrutivos não foram eficazes na diferenciação de efeitos deletérios existentes. Este estudo foi desenvolvido no âmbito do Programa de P\&D ANEEL, projeto 6491-0301/2013.
\end{abstract}

Palavras-chave: durabilide, enxofre, pirita, ensaios não destrutivos, ataque interno de sulfatos.

Abstract: This study evaluate the use of non destructive tests, ultrasound and electrical resistivity, in the differentiation of compounds contaminated by sulfide ions at three ages 84, 168 and 360 days. For this analysis four concrete series were molded, one being the reference, without contamination, and the others with a partial replacement of the small aggregate by pyrite $(0.38 \%, 0.75 \%$ and $3.75 \%)$. The contamination contents of the mixture were obtained from the normative regulations, which limit the content of $\mathrm{SO}_{3}$ with respect to the total weight of the aggregates of the compound. In order to favor the hydration reactions, it was decided to stay the test specimens, until 28 days, in a submerged cure with water saturated with lime. After this period, the materials were subjected to an accelerated aging mechanism, in which the specimens were alternated weekly between a tank with continuously aerated aqueous solution and a dry chamber, both with controlled temperature and humidity. Among the tests performed in this study, only the axial compressive strength showed a reduction in performance in the series of higher contamination. The intermediate series did not present inferior performance when compared to the reference series. This result corroborated to the normative recommendations, which allow the use of $0.5 \%(\mathrm{ACl})$ and $1.0 \%(\mathrm{AFNOR})$ of $\mathrm{SO}_{3}$ in relation to the total mass of aggregates. The results also showed that, for the proposed period and method of aging, the non destructive tests were not effective in the differentiation of deleterious effects. This study was developed under the ANEEL R\&D Program, project 6491-0301/2013.

Keywords: durability, sulfur, pyrite, non-destructive tests, internal attack of sulfates. 
CAPRARO, A. P. B.; ET AL., AVALIAÇÃO DE ENSAIOS NÃO DESTRUTIVOS PARA DETECÇÃO DE CONCRETOS DOSADOS COM DIFERENTES TEORES DE CONTAMINAÇÃO POR SULFETOS. $2^{\circ}$ Simpósio Paranaense de Patologia das Construções (20 SPPC), artigo 2SPPC1010, pp. 103-116, 2017. DOI: 10.4322/2SPPC.2017.010

\section{Introdução}

As estruturas de concreto participam da evolução histórica das obras de engenharia. Segundo Davood [1], apesar de a mais de um século o concreto ser utilizado na indústria da construção, a não mais que algumas décadas a durabilidade tem sido estudada por pesquisadores. De acordo com Fachinelli [2], os parâmetros de durabilidade estão ligados aos componentes do concreto, ao ambiente de exposição da estrutura e às características físico-químicas dos produtos gerados na hidratação do cimento. A degradação e a ruína de estruturas expostas a agentes agressivos como íons sulfato vêm atraindo a atenção de pesquisadores, em busca de melhorias na durabilidade do material [1], tema abordado no presente artigo.

Os mecanismos e as consequências do ataque por sulfato em estruturas de concreto apesar de amplamente estudados e discutidos ainda não estão integralmente entendidos [3]. Ademais, em se tratando, especificamente, do ataque interno por íons sulfato, proveniente da utilização de agregados contendo íons sulfeto, o seu mecanismo de atuação se faz necessário conhecer. O tema tem significância quanto ao entendimento dos processos de degradação do concreto quando instalados em grandes obras, principalmente, pela carência de técnicas e especificações normatizadas que dê diretrizes e limites sobre a utilização desse tipo de agregado, que em casos específicos, se faz necessário por questões logístico-financeiras, e até ambientais [4-5].

As reações ou os ataques internos por sulfato (ISA) têm sua origem nos agregados contaminados por sulfetos minerais, como por exemplo, a pirita, nome mais utilizado do dissulfeto de ferro ( $\mathrm{FeS}_{2}$ ), principal causador de reações físico-químicas nocivas ao concreto, nas adições minerais com íons sulfato e pelas soluções aquosas adsorvidas nos insumos [6-7]. Estas reações, tem caráter expansivo [2-8-9], gerando produtos até 2,5 vezes maiores que seus reagentes, causando tensões internas que podem ocasionar a fissuração do concreto, deteriorando o mesmo e abrindo caminhos para a penetração de outros agentes agressivos [10].

A pirita $\left(\mathrm{FeS}_{2}\right)$, quando em contato com a água e o oxigênio, se oxida. Esse processo pode gerar produtos como íons sulfato, que em contato com os produtos da hidratação do cimento como $\mathrm{Ca}(\mathrm{OH})_{2}$ e os produtos do cimento anidro como o $\mathrm{C}_{3} \mathrm{~A}$ (aluminato de cálcio) formam compostos mais volumosos, causando os efeitos deletérios nas estruturas de concreto [3-10]. Ainda, segundo os autores, é possível elencar os principais fatores que aceleram o processo de alteração dos sulfetos contidos nos agregados, sendo eles: a concentração elevada de oxigênio; a presença de umidade; a superfície específica; os altos teores de ferro e os valores de $\mathrm{pH}$ acima de 10 [3].

A importância do tema abordado é evidenciada em casos de ocorrência de manifestações patológicas geradas por esse tipo de ataque químico, relatados em diversos casos na literatura como por exemplo nas barragem de Torán [11], Grauss e Tabescán [12] situadas na Espanha. Outros exemplos foram observados na região de Trois-Rivières, em Quebec, no Canadá, onde mais de 400 moradores se depararam com degradações nas estruturas de concreto de suas residências, 2 a 4 anos após suas construções [13], e em alguns prédios e obras públicas construídos na década de 1970, na região de Barcelona, também na Espanha [14]. Já, no âmbito nacional, até então apenas alguns casos de contaminação de agregados foram alvos 
CAPRARO, A. P. B.; ET AL., AVALIAÇÃO DE ENSAIOS NÃO DESTRUTIVOS PARA DETECÇÃO DE CONCRETOS DOSADOS COM DIFERENTES TEORES DE CONTAMINAÇÃO POR SULFETOS. $2^{\circ}$ Simpósio Paranaense de Patologia das Construções (20 SPPC), artigo 2SPPC1010, pp. 103-116, 2017. DOI: 10.4322/2SPPC.2017.010

de pesquisas, dentre elas a Barragem do Rio Descoberto (Brasília/DF) [15] e a barragem de Irapé, localizada nas cidades de Grão Moró e Berilo (MG). Como relatado por Silva [16] "o conhecimento do problema de sulfetos é relativamente recente no Brasil e somente durante a construção da Usina Hidrelétrica de Irapé, onde o sulfeto se encontrava presente em altos teores na rocha de fundação, é que se passou a preocupar com esta possível ocorrência".

Tendo em vista o cenário exposto, o presente trabalho tem o objetivo avaliar o desempenho de duas técnicas não destrutivas, a de ultrassom e da resistividade elétrica do concreto, na diferenciação de concretos contaminados com diferentes teores de íons sulfeto. Para tanto, infere-se que seus resultados podem auxiliar no diagnóstico de estruturas anteriormente a quaisquer efeitos que venham a comprometer seu tempo operacional de projeto.

\section{Metodologia}

Para a avaliação do desempenho dos concretos com diferentes teores de contaminação foram adotadas quatro séries de estudo, sendo uma delas a referência, sem nenhuma contaminação, e outras três com diferentes teores de substituição do agregado miúdo por pirita.

Com o intuito de acompanhar o mecanismo de deterioração nos concretos estudados foram realizados três ensaios: o de resistência à compressão axial, o de ultrassom e o da resistividade elétrica do concreto, sendo os dois últimos ensaios não destrutivos e objeto de estudo desse trabalho.

Os ensaios foram realizados em três idades distintas dos concretos, sendo elas: 84, 168 e 360 dias, permitindo assim, o acompanhamento até um estágio avançado de hidratação dos compostos.

Para a realização dos ensaios foram moldados corpos de prova cilíndricos, de (10x20) $\mathrm{cm}$, seguindo as recomendações da NBR 5738 [17].

Até os 28 dias de idade, os corpos de prova foram mantidos em uma cura úmida saturada com cal. Após esse período, para a aceleração do mecanismo de degradação, foi adotado um procedimento semanal de aplicação alternada de diferentes parâmetros ambientais sobre os materiais, seguindo o princípio de algumas metodologias já empregadas por outros autores [18-19-20]. Neste caso, os corpos de prova foram submetidos a uma condição de imersão em um tanque com solução aquosa continuamente aerada por um sistema de borbulhamento e, alternadamente, a uma câmara seca com UR de $(55 \pm 5) \%$ e à temperatura média de $(23 \pm 2)^{\circ} \mathrm{C}$.

\subsection{Materiais}

Com relação aos aglomerantes optou-se pela composição do sistema cimentício em laboratório, substituindo-se $35 \%$ do CP V - ARI por cinza volante, de modo a classificar esta mistura de aglomerantes como cimento tipo CP IV de acordo com a NBR 5.736 (1991) [21]. O motivo foi trabalhar com uma classe de cimento similar ao CP IV que é muito utilizado em barragens de concreto, já que maior parte dos relatos de ocorrência do ataque por sulfato interno é neste tipo de estrutura. Não foi usado 
CAPRARO, A. P. B.; ET AL., AVALIAÇÃO DE ENSAIOS NÃO DESTRUTIVOS PARA DETECÇÃO DE CONCRETOS DOSADOS COM DIFERENTES TEORES DE CONTAMINAÇÃO POR SULFETOS. $2^{\circ}$ Simpósio Paranaense de Patologia das Construções (2० SPPC), artigo 2SPPC1010, pp. 103-116, 2017. DOI: 10.4322/2SPPC.2017.010

diretamente um cimento CP IV comercial porque, neste caso, não se tem a informação do teor de pozolana do aglomerante.

O cimento CP V - ARI empregado possuía uma massa específica de $3,13 \mathrm{~g} / \mathrm{cm}^{3}$ e uma área específica BET de 1,07 m²/g. Já, a cinza volante, proveniente da combustão do carvão mineral da jazida do vale do rio Tubarão, apresentou uma massa específica de $1,95 \mathrm{~g} / \mathrm{cm}^{3}$ e uma área específica BET de 1,09 $\mathrm{m}^{2} / \mathrm{g}$.

O agregado miúdo empregado foi uma areia natural, proveniente da região de São Luiz do Purunã, no Paraná. As características físicas principais do material como: a massa específica; a massa unitária; o teor de material pulverulento; e o módulo de finura; foram obtidas com base nas recomendações normativas e estão apresentados na Tabela 1.

Tabela 1: Resultados analíticos dos testes de caracterização do agregado miúdo, areia natural utilizada nas dosagens de concreto desta pesquisa

\begin{tabular}{lcl}
\hline \multicolumn{1}{c}{ Propriedade } & $\begin{array}{c}\text { Valores obtidos } \\
\text { no teste }\end{array}$ & \multicolumn{1}{c}{ Norma técnica } \\
\hline Massa Específica, g/cm & 2,38 & NBR NM $52(2009)[22]$ \\
Massa Unitária, g/cm ${ }^{3}$ & 1,496 & NBR NM 45 (2006) [23] \\
Teor de pulverulentos, \% & 10,13 & NBR NM 46 (2003) [24] \\
Módulo de finura & 1,41 & NBR NM 248 (2003) [25] \\
\hline
\end{tabular}

O agregado graúdo utilizado foi uma brita 1, proveniente da região de Curitiba, Paraná. O material foi um basalto com diques de diabásio e suas características físicas estão apresentadas na Tabela 2.

Nenhum dos agregados empregados possuiu, em sua composição, a contaminação natural pelo íon sulfeto. Dessa forma, optou-se pela contaminação artificial do agregado miúdo, substituindo parte de sua massa por pirita.

A pirita utilizada foi proveniente da região de Figueira, no Paraná. Esse material foi obtido de um resíduo do processo de beneficiamento do carvão mineral. Nos ensaios de caracterização o material apresentou massa específica igual a 4,95 g/cm³ e, após o seu processo de moagem, foi classificada como um material passante pela peneira $2,4 \mathrm{~mm}$.

Tabela 2: Resultados analíticos dos testes de caracterização do agregado graúdo, utilizado nas dosagens de concreto desta pesquisa

\begin{tabular}{lcc}
\hline \multicolumn{1}{c}{ Propriedade } & $\begin{array}{c}\text { Valores } \\
\text { obtidos } \\
\text { no teste }\end{array}$ & Norma técnica \\
\hline Massa Específica, g/cm & 2,72 & NBR NM $53(2009)[26]$ \\
Massa Unitária, g/cm $\mathrm{cm}^{3}$ & 1,625 & NBR NM $45(2006)[23]$ \\
Absorção, \% & 0,98 & NBR NM $53(2009)[26]$ \\
Dimensão máxima característica, mm & 19,10 & NBR NM 248(2003) [25] \\
Módulo de finura & 6,80 & NBR NM 248 (2003) [25] \\
\hline
\end{tabular}




\subsection{Moldagens}

Os corpos de prova cilíndricos foram moldados conforme as recomendações da NBR 5738 [17].

O traço adotado como referência (traço médio de concreto convencional empregado em usina hidroelétrica nacional) foi de 1:2,70: 2,70 e a relação água/cimento de 0,60.

Para este estudo foram estabelecidos 4 teores de contaminação do material por $\mathrm{SO}_{3}$, com base no que preconiza a ACl 201.2R-01 (1991) [27], sendo dois teores abaixo do limite de norma: o de referência; e com 0,5\%, em massa, de $\mathrm{SO}_{3}$; e 2 teores acima desde limite: um contendo $1 \%$, em massa, de $\mathrm{SO}_{3}$; e outro com $5 \%$, em massa, deste mesmo contaminante. Neste artigo os traços estão nomeados como $0,0 \% \mathrm{SO}_{3}$; $0,5 \% \mathrm{SO}_{3} ; 1,0 \% \mathrm{SO}_{3}$ e $5,0 \% \mathrm{SO}_{3}$.

Destaca-se que a norma francesa NF P18-540 (1997) [28], que permite a utilização de agregados contaminados, desde que o teor não ultrapasse $1 \%$ da massa total de agregados.

Como no estudo foi empregada a pirita como contaminante, foi necessária a multiplicação dos teores por um fator proporcional em quantidade de enxofre entre os dois compostos. Na Tabela 3, estão apresentados os teores de contaminação empregados, em $\mathrm{SO}_{3}$ e $\mathrm{FeS}_{2}$.

Tabela 3: Teores de contaminação das dosagens pelo $\mathrm{SO}_{3}$, baseados nas proporções equivalentes de pirita adicionada

\begin{tabular}{cc}
\hline Teor de $\mathrm{SO}_{3}$ & Teor de pirita \\
\hline $0,0 \%$ & $0,00 \%$ \\
$0,5 \%$ & $0,38 \%$ \\
$1,0 \%$ & $0,75 \%$ \\
$5,0 \%$ & $3,75 \%$ \\
\hline
\end{tabular}

As porcentagens de contaminação foram aplicadas sobre a massa total de agregados e a substituição foi feita com o agregado miúdo, material com granulometria similar à pirita.

A avaliação do ataque interno por sulfatos foi realizada em três idades dos concretos: 84, 168 e 360 dias. Em cada uma delas, foram executados três ensaios: o de resistência à compressão axial, o da velocidade de propagação de ondas de ultrassom e o da resistividade elétrica do concreto.

Portanto, para este estudo, no total foram moldados 60 corpos de prova, sendo 5 para cada traço, em cada idade.

\subsection{Ensaios}

Um dos ensaios realizados nos corpos de prova cilíndricos foi o de resistência à compressão axial. Os corpos de prova cilíndricos foram ensaiados em conformidade à NBR 5739 (2007) [29]. 
CAPRARO, A. P. B.; ET AL., AVALIAÇÃO DE ENSAIOS NÃO DESTRUTIVOS PARA DETECÇÃO DE CONCRETOS DOSADOS COM DIFERENTES TEORES DE CONTAMINAÇÃO POR SULFETOS. $2^{\circ}$ Simpósio Paranaense de Patologia das Construções (2० SPPC), artigo 2SPPC1010, pp. 103-116, 2017. DOI: 10.4322/2SPPC.2017.010

Para que a planicidade entre as faces fosse garantida, antes do ensaio, as amostras foram retificadas. A máquina de ensaio utilizada foi uma prensa Dinatest, utilizando uma velocidade de carregamento na faixa de $(0,45 \pm 0,15) \mathrm{MPa} / \mathrm{s}$, como estabelecido na NBR 5739 (2007) [29].

Outro ensaio, realizado nos corpos de prova nas idades pré-determinadas, foi o de ultrassom, conforme a NBR 8802 (2013) [30], que tem por objetivo determinar a velocidade de propagação de ondas no interior dos corpos de prova cilíndricos.

Por fim, foi aplicado o ensaio de resistividade elétrica pelo Método Wenner. O ensaio foi realizado para definir uma importante propriedade do concreto, a capacidade do concreto em resistir à passagem de corrente elétrica, e está relacionada a sua permeabilidade e a sua difusividade aos íons através dos poros do concreto [31]. $O$ ensaio consistiu no posicionamento de quatro eletrodos na superfície do concreto. Os mesmos foram alinhados e estiveram posicionados equidistantes um do outro. Como o ensaio foi realizado em corpos cilíndricos de $(10 \times 20) \mathrm{cm}$, foi aplicado um coeficiente de fator de forma de 0,377, como é recomendado pela UNE 83988-2 (2012) [32].

\subsection{Análise dos resultados}

Os resultados obtidos com os ensaios propostos no programa experimental foram tratados estatisticamente por meio do teste de Tukey, com 95\% de confiança. Além disso, foram consideradas válidas as leituras obtidas quando os valores individuais não diferiram da média em mais de 10\%.

\section{Resultados e discussões}

Neste item, estão apresentados e discutidos os resultados dos três ensaios realizados: resistência à compressão axial, ultrassom e de resistividade elétrica do concreto.

Na Figura 1, estão mostrados os resultados médios da resistência à compressão axial em função do teor de contaminação por $\mathrm{SO}_{3}$ das dosagens e das idades de envelhecimento de 84,168 e de 360 dias. Na primeira idade avaliada, aos 84 dias, foi notada equivalência estatística entre as séries de 0,0\% $\mathrm{SO}_{3}$ e $0,5 \% \mathrm{SO}_{3}$, bem como entre as séries de $1,0 \% \mathrm{SO}_{3}$ e $5,0 \% \mathrm{SO}_{3}$. Considerando a análise estatística pode se afirmar que, nesta idade, as amostras com teores de 1,0\% $\mathrm{SO}_{3}$ e $5,0 \% \mathrm{SO}_{3}$ apresentaram resistências inferiores (cerca de $20 \%$, em média), quando comparadas com as de teores menores.

Hasparyk et al. (2003) [9] encontraram resistências inferiores (cerca de 10\%), na idade de 91 dias, para a série contaminada com 1,0\% $\mathrm{SO}_{3}$, quando esta foi comparada com a referência. No entanto, os autores concluíram que os concretos não foram significativamente afetados, neste período, pela presença dos sulfetos presentes. A diferença observada entre os valores finais da propriedade já aos 84 dias deste trabalho e o da Hasparyk et al. (2003) [9], foi atribuída aos ciclos alternados de secagem e molhagem, diferentemente ao de exposição direta em câmara úmida, deste último, permitindo, no processo adotado neste trabalho, diferentes teores de oxigenação dos corpos de prova e, consequentemente, a aceleração da sua degradação. 
Analisando comparativamente os resultados das idades de 84 e 168 dias notou-se um aumento significativo de resistência em todos os casos. Este, foi mais acentuado para a série $1,0 \% \mathrm{SO}_{3}$ (cerca de $85 \%$ ) e mais discreto, em termos de porcentagem, para a série de menor contaminação, $0,5 \% \mathrm{SO}_{3}$ (cerca de $28 \%$ ). Entretanto, considerando-se o ganho de resistência em valores absolutos, o traço que menos ganhou entre as idades de 84 e 168 dias, foi o com teor de 5,0\% $\mathrm{SO}_{3}$.

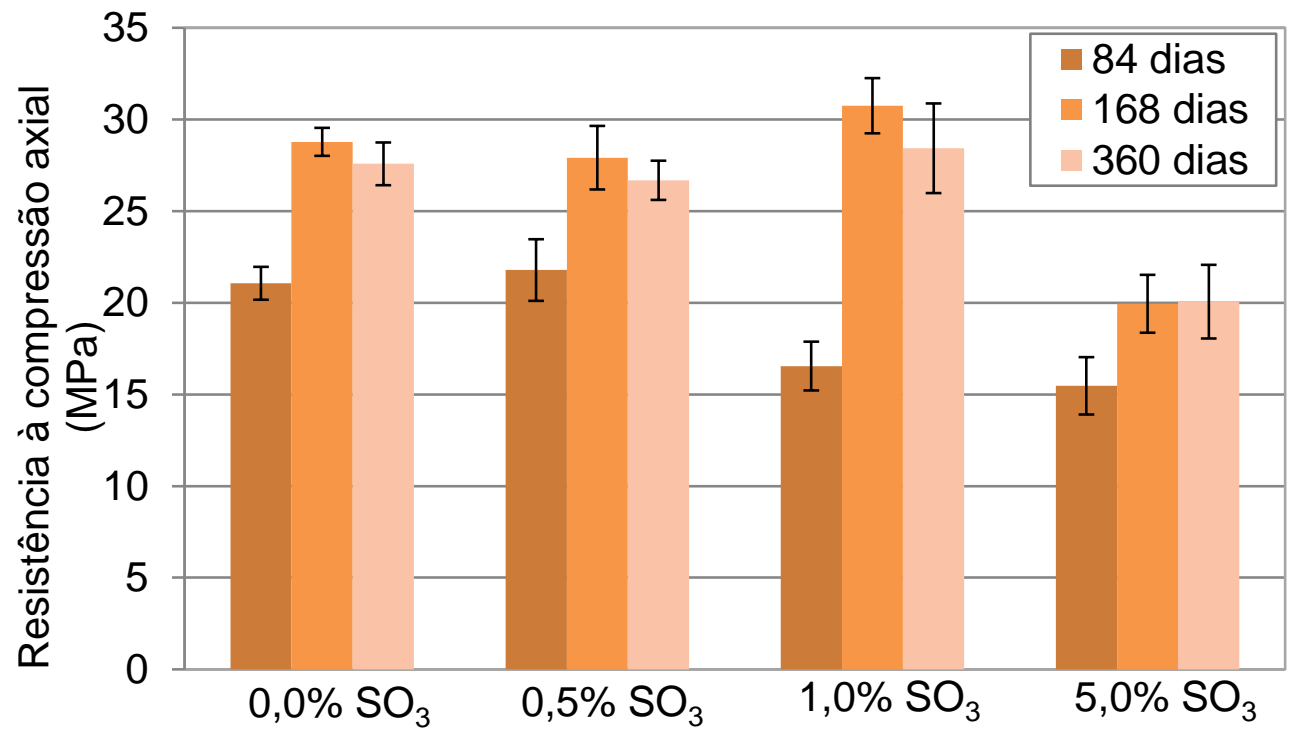

Figura 1: Resultados da resistência à compressão axial das séries nas três idades estudadas (condição acelerada de molhagem e secagem).

O aumento da resistência de séries contaminadas nas primeiras idades, já foi observado em outros trabalhos e isso pode ser justificado pela colmatação dos poros pelos produtos originários do ataque interno de sulfatos [33-34-35].

O menor ganho de resistência ocorrido para o maior teor de contaminação estudado $\left(5,0 \% \mathrm{SO}_{3}\right)$, pode estar relacionado ao aumento das tensões internas do concreto pelo aumento do seu bulk, ou mesmo, reações químicas paralelas entre o sulfato e os componentes da pasta cimentícia, ambos, não estudados no presente trabalho.

$\mathrm{Na}$ idade de 168 dias a série que obteve a maior resistência a compressão foi a de $1,0 \% \mathrm{SO}_{3}$ e a menor resistência foi a de $5,0 \% \mathrm{SO}_{3}$. As séries $0,0 \% \mathrm{SO}_{3}$ e $0,5 \% \mathrm{SO}_{3}$ continuaram a apresentar resistências equivalentes.

Se comparado aos resultados com idade de 168 dias, aos 360 dias de idade, 0 aumento de resistência não foi significativo para nenhuma das séries estudadas. Nesta idade, a série $1,0 \% \mathrm{SO}_{3}$ continuou apresentando as maiores resistências a compressão, seguida das séries $0,0 \% \mathrm{SO}_{3}$ e $0,5 \% \mathrm{SO}_{3}$ (equivalentes) e por fim a série de maior contaminação $5,0 \% \mathrm{SO}_{3}$.

A Figura 2 apresenta a velocidade de ultrassom em função da evolução do tempo para a série de referência e as séries com os três teores de contaminação por $\mathrm{SO}_{3}$ deste estudo.

Pelos resultados da velocidade de propagação de ondas ultrassônicas aos 84 dias, inferiu-se estatisticamente a equivalência entre as séries $0,0 \% \mathrm{SO}_{3}, 0,5 \% \mathrm{SO}_{3}$ e $5,0 \%$ 
CAPRARO, A. P. B.; ET AL., AVALIAÇÃO DE ENSAIOS NÃO DESTRUTIVOS PARA DETECÇÃO DE CONCRETOS DOSADOS COM DIFERENTES TEORES DE CONTAMINAÇÃO POR SULFETOS. $2^{\circ}$ Simpósio Paranaense de Patologia das Construções (2० SPPC), artigo 2SPPC1010, pp. 103-116, 2017. DOI: 10.4322/2SPPC.2017.010

$\mathrm{SO}_{3}$ (Figura 2). Enquanto a série $1,0 \% \mathrm{SO}_{3}$ só pode ser considerada equivalente à série $0,5 \% \mathrm{SO}_{3}$.

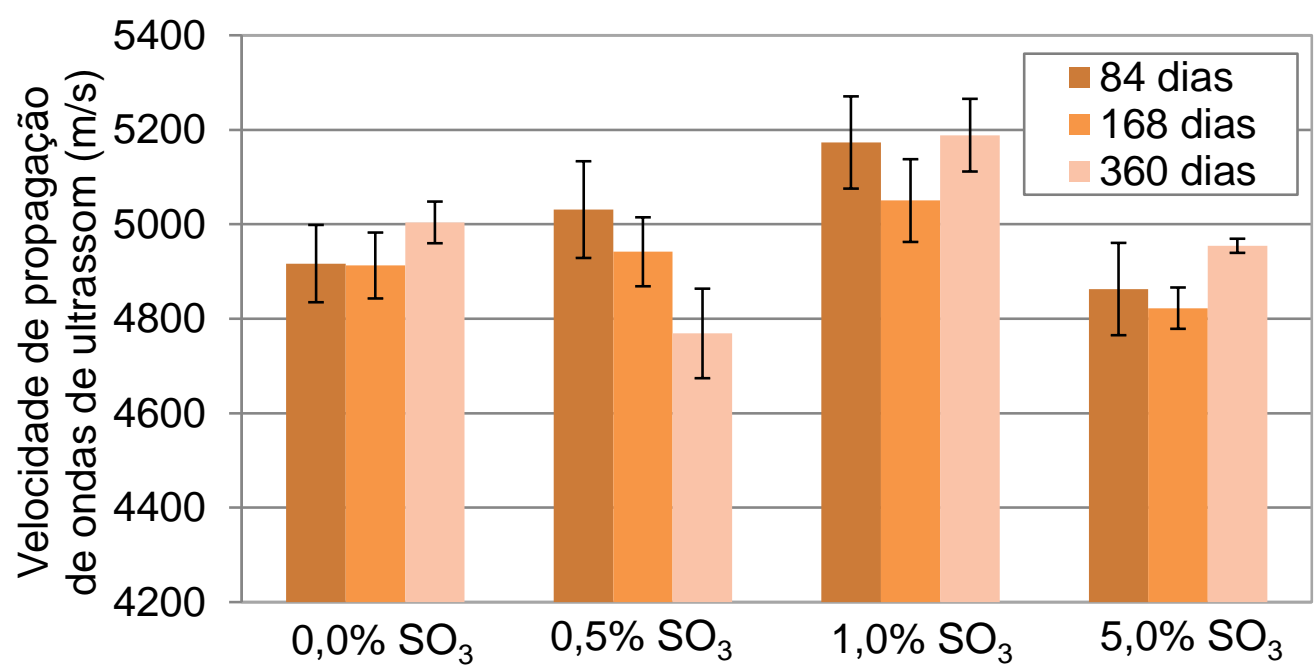

Figura 2: Velocidade de propagação de ondas de ultrassom das séries nas três idades estudadas (condição acelerada de molhagem e secagem)

Aos 168 e 360 dias de idade não foi notado estatisticamente o aumento ou redução dos valores para nenhuma série, quando aplicado o teste Tukey, comparando-se aos valores dos 84 dias.

Analisando os resultados com base na NBR 8802 (2013) [30], notou-se que todas as séries avaliadas se enquadraram na classificação da qualidade do concreto como excelente (>4500 m/s), ou seja, pela metodologia empregada não se pode distinguir o efeito da contaminação ou do ataque por íons sulfato nas amostras testadas, no tempo de exposição e com o processo de envelhecimento empregados.

Na Figura 3 estão apresentados os dados de resistividade elétrica superficial obtidos para a série de referência e para as séries com os três teores de contaminação deste estudo.

Pelos resultados obtidos verificou-se que na idade de 84 dias somente a série com maior contaminação, $5,0 \% \mathrm{SO}_{3}$, apresentou resultados estatisticamente diferentes das demais, sendo seus valores inferiores.

Já aos 168 dias, as séries $0,0 \% \mathrm{SO}_{3}, 0,5 \% \mathrm{SO}_{3}$ e 5,0\% $\mathrm{SO}_{3}$ puderam ser consideradas estatisticamente equivalentes e a série de $1,0 \% \mathrm{SO}_{3}$ apresentou a maior resistividade nesta idade.

Por fim, aos 360 dias, a série de referência $\left(0,0 \% \mathrm{SO}_{3}\right)$ foi a que apresentou a maior resistividade, sendo as demais séries (contaminadas) consideradas equivalentes.

Observou-se, adicionalmente, que diferente do que ocorreu no ensaio de resistência à compressão e de velocidade de pulso de ultrassom, as medidas de resistividade não indicaram tendência de estabilização mesmo a idades mais avançadas, como 168 e 360 dias. Isso pode ser considerado um indicativo que existem mudanças 
CAPRARO, A. P. B.; ET AL., AVALIAÇÃO DE ENSAIOS NÃO DESTRUTIVOS PARA DETECÇÃO DE CONCRETOS DOSADOS COM DIFERENTES TEORES DE CONTAMINAÇÃO POR SULFETOS. $2^{\circ}$ Simpósio Paranaense de Patologia das Construções (2० SPPC), artigo 2SPPC1010, pp. 103-116, 2017. DOI: 10.4322/2SPPC.2017.010

químicas ocorrendo no interior dos concretos em análise e seus efeitos mecânicos podem se manifestar posteriormente.

Uma possível análise da resistividade elétrica do concreto é atrelada a probabilidade de corrosão do composto [36]. Os resultados expostos mostraram que a série sem contaminação, $0,0 \% \mathrm{SO}_{3}$, possuiu a maior resistividade aos 360 dias de idade $(40,2$ $\mathrm{k} \Omega . \mathrm{cm}$ ). A diferença obtida entre essa série e as demais foi próxima a $30 \%$ em todos os casos. Contudo, quando feita a análise dos resultados segundo a classificação citada por Cascudo (1997) [36], todas as séries foram classificadas como de probabilidade de corrosão desprezível (> $20 \mathrm{k} \Omega . \mathrm{cm})$.

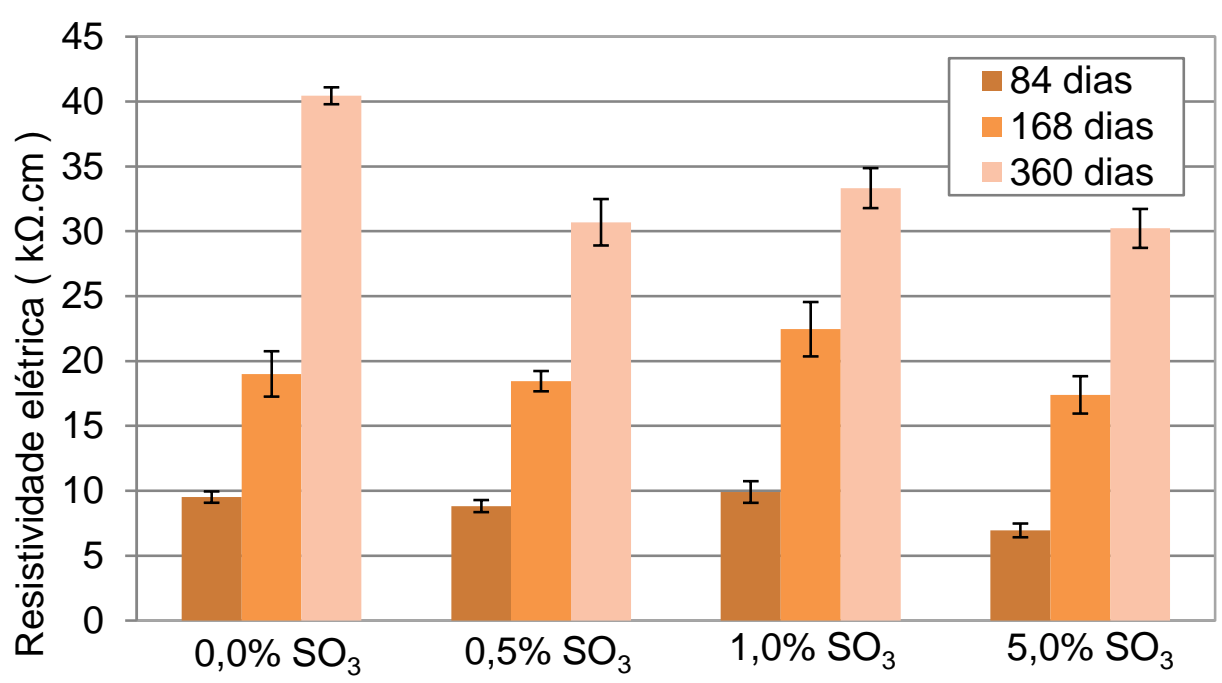

Figura 3: Resistividade elétrica do concreto das séries nas três idades estudadas (condição acelerada de molhagem e secagem)

Outra análise feita para avaliar a eficiência dos ensaios na diferenciação de compostos contaminados com diferentes teores foi a de correlação linear entre os resultados dos ensaios nas idades estudadas e os respectivos teores. O ensaio que apresentou maior correlação linear foi o de resistência à compressão axial (Figura 4), com coeficiente de determinação $\left(R^{2}\right)$ de 0,9032 , aos 360 dias de idade. Notou-se, adicionalmente, que todas as equações obtidas apresentaram coeficientes angulares negativos, indicando o decréscimo de resistência conforme aumentados os teores de contaminação. 


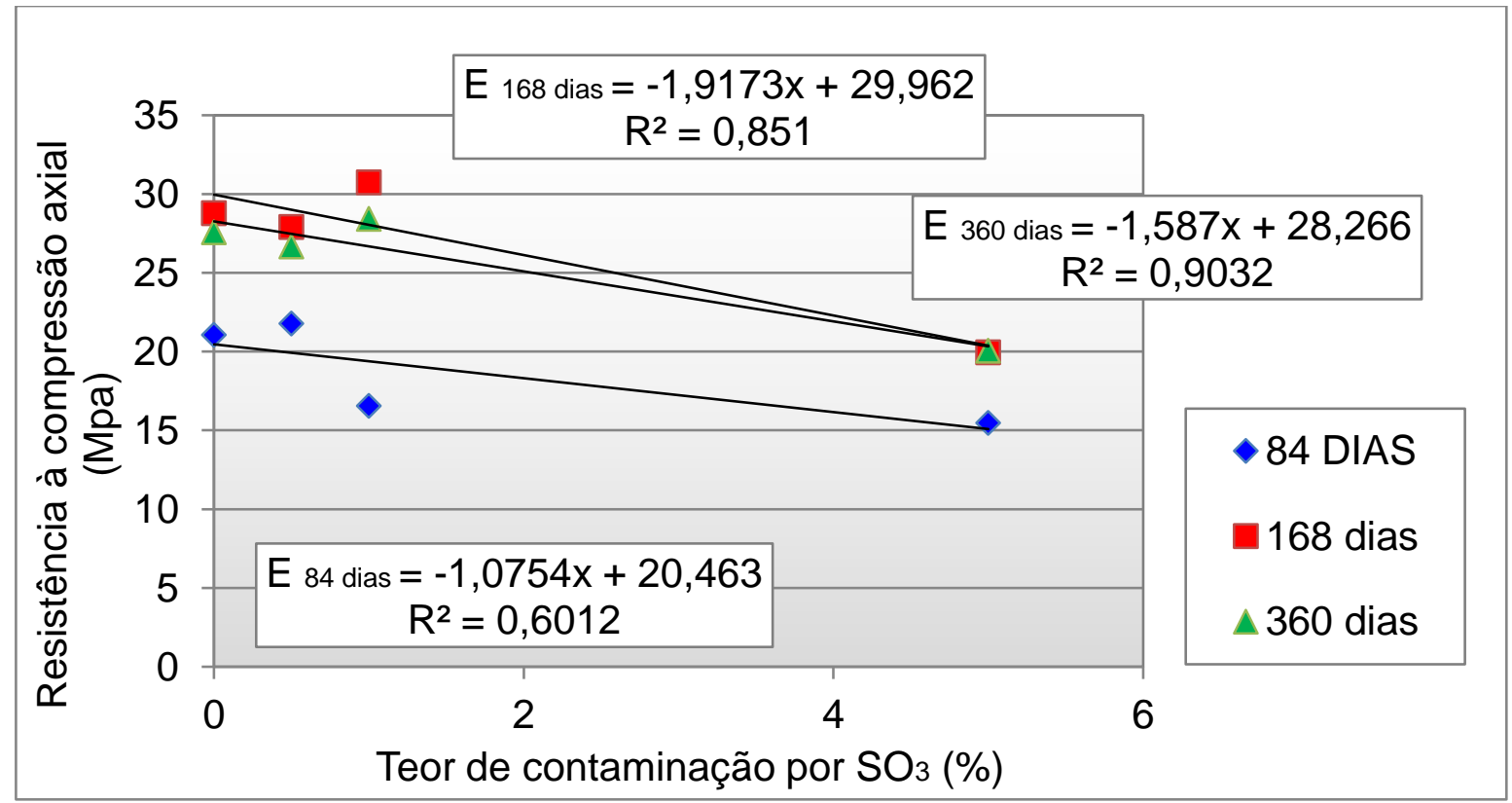

Figura 4: Resultados estatísticos da correlação entre o ensaio de resistência à compressão axial e os teores de contaminação empregados

O ensaio não destrutivo que apresentou maiores coeficientes de correlação linear foi o de resistividade elétrica do concreto, como pode ser observado na Figura 5. Neste caso, a maior correlação ocorreu na idade de 84 dias, com coeficiente de determinação $\left(R^{2}\right)$ igual a 0,815. Para as demais idades as correlações puderam ser consideradas baixas, no entanto, o comportamento decrescente, indicado pelos coeficientes angulares negativos, também pode ser observado em todas as idades, semelhante ao ensaio de resistência à compressão axial.

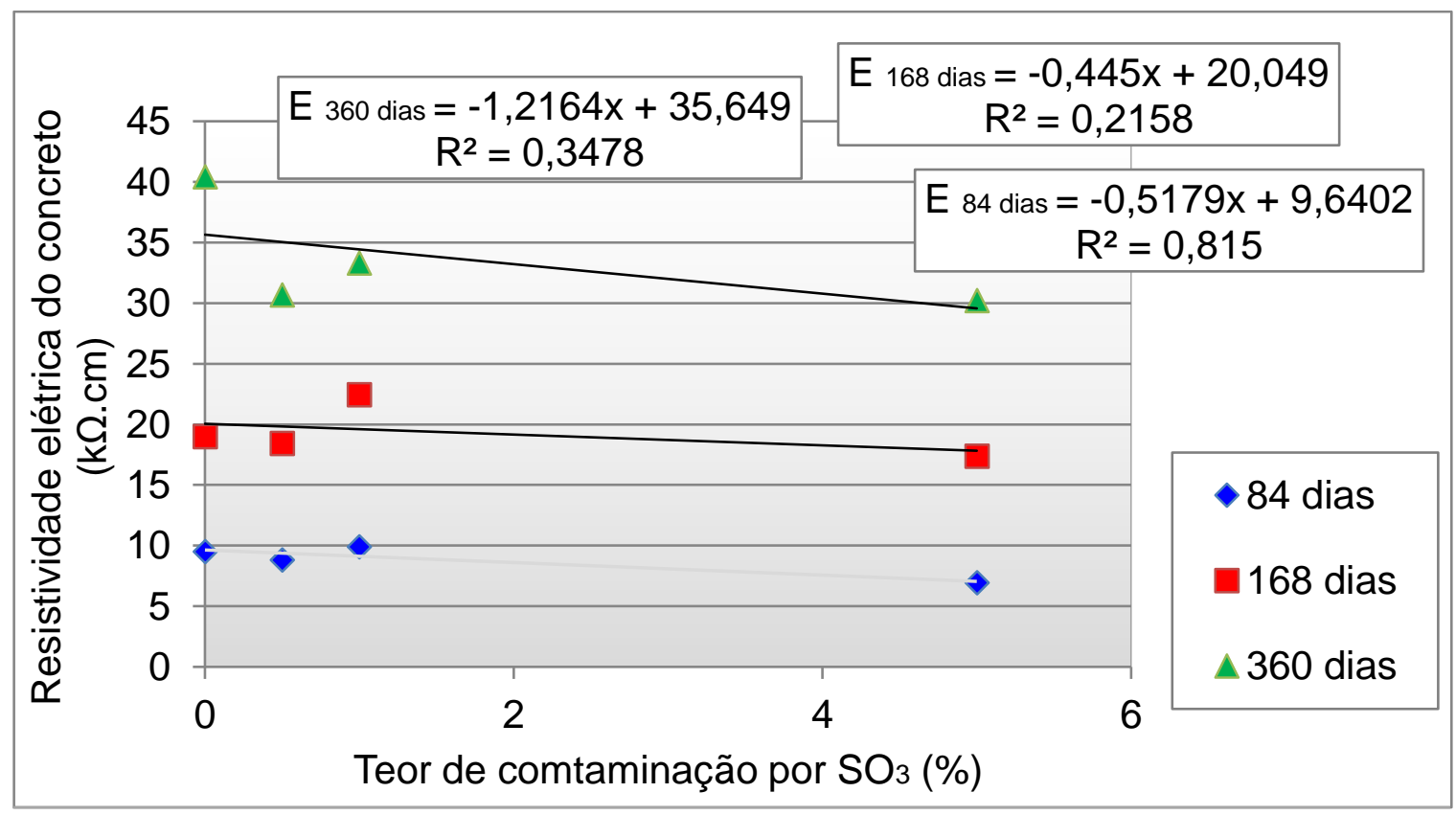

Figura 5: Resultados estatísticos da correlação entre o ensaio de resistividade elétrica do concreto e os teores de contaminação empregados

Pelo ensaio de ultrassom se obtiveram as correlações mais baixas e, também, descrescentes para as idades de 84 e 360 dias (Figura 6). 


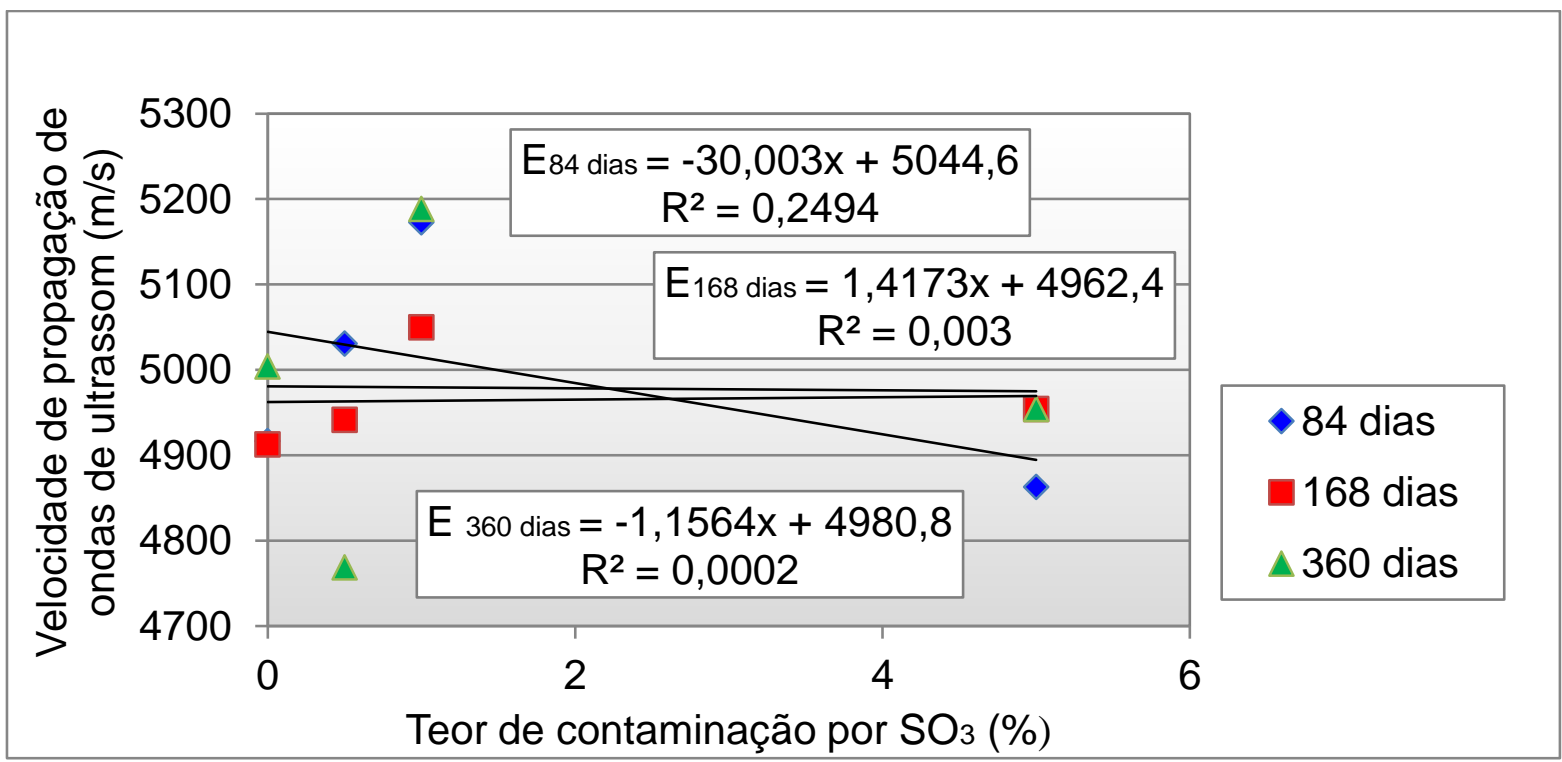

Figura 6: Resultados estatísticos da correlação entre o ensaio de Velocidade de propagação de ondas de ultrassom e os teores de contaminação empregados

\section{Conclusões}

Dentre os ensaios realizados neste estudo, somente o de resistência à compressão axial apresentou redução do desempenho na série de maior contaminação. As séries intermediárias $\left(0,5 \% \mathrm{SO}_{3}\right.$ e $\left.1,0 \% \mathrm{SO}_{3}\right)$, no período deste estudo, não apresentaram desempenho inferior quando comparadas à série referência. Este resultado corrobora com as recomendações normativas, as quais permitem a utilização de $0,5 \%(\mathrm{ACl})$ e $1,0 \%$ (AFNOR) de $\mathrm{SO}_{3}$ com relação à massa total de agregados.

Pelos resultados do ensaio de resistividade elétrica, na idade de 84 dias e com o método de envelhecimento estudado, pode-se inferir pela sua capacidade em diferenciar as séries contaminadas da referência, pois apresentou resultados com coeficiente de determinação $\left(R^{2}\right)$ igual a 0,815 .

Pelas diferenças estatísticas obtidas nos dois ensaios não destrutivos (velocidade de ultrassom e resistividade elétrica), pode-se considerar que não foram consideradas técnicas eficazes para se atribuir quaisquer efeitos deletérios nos corpos de prova de concreto, pelos contaminantes, seus teores adicionados, pelo tempo e tipo de envelhecimento adotados.

Conclui-se, ainda, que, a fim de melhorar a investigação da eficácia destes ensaios não destrutivos na avaliação da degradação dos materiais, utilizando-se as mesmas técnicas adotadas neste estudo, há necessidade de estudá-los para idades maiores.

\section{Agradecimentos}

Os autores agradecem à infraestrutura e ao apoio em recursos humanos e financiamento à COPEL GeT, projeto P\&D 6491-0301/2013, à ANEEL, à 
CAPRARO, A. P. B.; ET AL., AVALIAÇÃO DE ENSAIOS NÃO DESTRUTIVOS PARA DETECÇÃO DE CONCRETOS DOSADOS COM DIFERENTES TEORES DE CONTAMINAÇÃO POR SULFETOS. $2^{\circ}$ Simpósio Paranaense de Patologia das Construções (2० SPPC), artigo 2SPPC1010, pp. 103-116, 2017. DOI: 10.4322/2SPPC.2017.010

Coordenação de Aperfeiçoamento de Pessoal de Nível Superior (CAPES), à Fundação Araucária, aos Institutos LACTEC, ao CNPq Lei 8010/90 (LI 15/21872141; LI 14/4695814-5; LI14/3410726-9), ao CNPq, bolsa DT, processo, 302672/2016-8 e a Universidade Federal do Paraná - PPGECC/UFPR.

\section{Referências}

[1] Davood M., Farzaneh N., Hamed N.-M. (2016). Influence of magnesium sulphate concentration on durability of concrete containing micro-silica, slag and limestone powder using durability index, Construction and building, 117:107-120.

[2] Faquinelli, C.R. (2010). Estudo da reatividade de agregados contendo sulfetos em concretos e argamassas. Dissertação (mestrado), Universidade Federal de Minas Gerais - UFMG, Belo Horizonte, Brasil.

[3] Depuy, G. W. (1994). Chemical resistance of concrete, Significance of tests and properties of Concrete and concrete making materials, ASTM STP 169C, Philadelphia, Estados Unidos da América.

[4] Gomides, M.; Hasparyk, N.; Andrade, M.; Bittencourt, R. (2005). Estudo em laboratório de concretos contendo agregados com sulfetos, In: Congresso Brasileiro do Concreto, 47 CBC, , Recife, Brasil.

[5] Gomides, M. (2009) Investigação de agregados contendo sulfetos e seus efeitos sobre a durabilidade do concreto. Tese (Doutorado), Universidade Federal do Rio Grande do Sul - UFPR, Porto Alegre, Brasil.

[6] Pereira, E.; Portella, K.F.; Bragança, M.O.G. P.; Ochiliski, L. M.; Oliveira, I. C. (2016). Oxidação da pirita e seus efeitos em argamassas de cimento Portland sujeitas ao ataque por sulfatos de origem interna. Revista Matéria, 11705: 342354.

[7] Neville, A. (2004). The confused world of sulfate attack on concrete, Cement and concrete research, 34: 1275-1296.

[8] Tagnit-Hamou, A.; Saric-Coric, M.; Rivard, P. (2005). Internal deterioration of concrete by the oxidation of pyrrhotitic agregates, Cement and concrete research, 35: 99-107.

[9] Hasparik, N. P., Nascimento, J. F. F., Andrade, M. A. S., et al., Estudos de laboratório com concretos contendo agregados obtidos a partir de rocha com sulfetos, In: Reunión Técnica de la AATH - Seminario sobre Hormigones Especiales, 15므, Argentina, 2003.

[10] Coutinho, J. S. (2001). Ataque por sulfatos. Faculdade de Engenharia da Universidade do Porto. Porto, Portugal.

[11] Ayora, C.; Chinchón, S.; Aguado, A.; Guirado, F. (1998). Weathering of iron sulfides and concrete alteration: thermodynamic model and observation in dams from central pyrenees, spain. Cement and concrete research, 28: 591 603.

[12] Mieza, J. A. (1998). Integrated studies of the behaviour of the Graus and Tabescán dams. Dam Safety. Proceedings of International Symposium on New 
Trends and Guidelines on Dam Safety. Volume 1, ed. La Berga, Barcelona, Espanha.

[13] Duchesne, J.; Fournier, B. (2013). Deterioration of Concrete by the Oxidation of Sulphide Minerals in the Aggregate. Journal of Civil Engineering and Architecture, 7: 922-931.

[14] Ayora, C.; Chinchón, S.; Aguado, A.; Guirado, F. (2015). Influence of weathering of iron sulfides contained in aggregates on concrete. Cement ad Concrete. Research, 25: 1264-1272.

[15] Correa, M.F. (2001). Sistema de recuperação do maciço da barragem do rio Descoberto", Dissertação (Trabalho de conclusão de curso) , Universidade de Brasília, Brasília, Brasil.

[16] Silva, H.H.A.B., Graça, N.G., Ribeiro, E.E.W.A., Gomides, M.J. (2007). Metodologias para Investigação de Sulfetos em Agregados para Concreto Experiências de Furnas utilizadas em Obras - Congresso Brasileiro do Concreto, 49 CBC, Bento Gonçalves, Rio Grande do Sul, Brasil.

[17] NBR 5738 (2015) Concreto - Procedimento para moldagem e cura de corpos de prova. Brasileira de Normas Técnicas - ABNT, Rio de Janeiro, Brasil.

[18] Gao, J.; Yu, Z.; Song, L.; Wang, T.; Wei, S. (2013). Durability of concrete exposed to sulfate attack under flexural loading and drying-wetting cycles. Construction and Building Materials, 39: 33-38.

[19] Gong, J.; Cao, J.; Wang, Y. (2016). Effects of sulfate attack and dry-wet circulation on creep of fly-ash slag concrete. Construction and Building Materials, 125:12-20

[20] Jiang, L.; Niu, D. (2016). Study of deterioration of concrete exposed to different types of sulfate solutions under drying-wetting cycles, Construction and Building Materials, 117: 88-98.

[21] NBR 5736 (1991). Cimento Portland pozolânico. Brasileira de Normas Técnicas - ABNT, Rio de Janeiro, Brasil.

[22] NBR NM 52 (2009). Agregado miúdo - Determinação da massa específica e massa específica aparente. Associação Brasileira de Normas Técnicas ABNT, Rio de Janeiro, Brasil.

[23] NBR NM 45 (2006). Agregados - Determinação da massa unitária e do volume de vazios. Associação Brasileira de Normas Técnicas - ABNT, Rio de Janeiro, Brasil.

[24] NBR NM 46 (2003). Agregados - Determinação do material fino que passa através da peneira 75 um, por lavagem. Associação Brasileira de Normas Técnicas - ABNT, Rio de Janeiro, Brasil.

[25] NBR NM 248 (2003). Agregados - Determinação da composição granulométrica. Associação Brasileira de Normas Técnicas - ABNT, Rio de Janeiro, Brasil.

[26] NBR NM 53 (2009). Agregado graúdo - Determinação da massa específica, massa específica aparente e absorção de água. Associação Brasileira de Normas Técnicas - ABNT, Rio de Janeiro, Brasil.

[27] ACI 201 (1991) Guide to durable concrete. American Concrete Institute - ACI, Detroit, Estados Unidos da América. 
[28] XP P18-540 (1997). Granulats - Définitions, conformité, spécifications. Association Française de Normalisation - AFNOR, Paris, França.

[29] NBR 5739 (2007). Concreto - Ensaios de compressão de corpos de prova cilíndricos. Associação Brasileira de Normas Técnicas - ABNT, Rio de Janeiro, Brasil.

[30] NBR 8802 (2013). Concreto endurecido - Determinação da velocidade de propagação de onda ultrassônica. Associação Brasileira de Normas Técnicas - ABNT, Rio de Janeiro, Brasil.

[31] Whiting, D. A.; Nagi, M. A. (2003). Eletrical resistivity of concrete - A literature review. (R\&D Serial No. 2457) Portland Cement Association, Skokie, Illinois, Usa, 2003.

[32] UNE 83988-2 (2012) Durabilidad del hormigón - determinación de la resistividad - Parte 2: Método de las cuatro puntas o de Wenner. Associación Española de Normalización y Certificación - AENOR, Madri, Espanha.

[33] Biczok, I. "Concrete Corrosion and Concrete Protection." Chemical Publishing Company, Inc., New York, 1972.

[34] Ouyang, W.; chen, J.; Jiang, M. (2014). "Evolution of surface hardness of concrete under sulfate attack." Construction and Building Materials, Vol. 53, p. $419-424$

[35] Oliveira, I. C. "Análises de dados para a elaboração de diretrizes visando à detecção de sulfetos e sulfatos na composição de CCR." Dissertação mestrado, Universidade Federal do Paraná. Curitiba, 2013.

[36] Cascudo, O. M. O controle da corrosão de armaduras de concreto. PINI. São Paulo, 1997. 